'Universidad Viña del Mar, Chile. ¿Universidad Autónoma de Chile. ${ }^{3}$ Laboratorio de Motricidad Humana, Escuela de Educación Física, Facultad de Filosofía y Educación Laboratorio de Antropología Física y Anatomía Humana, Instituto de Biología, Facultad de Ciencias. Pontificia Universidad Católica de Valparaíso, Chile. ${ }^{a}$ GICEFYD, Grupo de Investigación en Ciencias del Deporte. Carrera en Pedagogía en Educación Física.

Recibido el 20 de agosto de 2012, aceptado el 16 de enero de 2013

Correspondencia a:

Dr. Carlos Cristi Montero Facultad de Ciencias de la Salud Universidad Autónoma de Chile. Carlos Antúnez 1920,

Providencia, Santiago, Chile. carlos.cristi.montero@gmail.com

\section{Paradoja: "activo físicamente pero sedentario, sedentario pero activo físicamente". Nuevos antecedentes, implicaciones en la salud y recomendaciones}

\author{
CARLOS CRISTI-MONTERO Phd ${ }^{1,2 a}$, FERNANDO RODRÍGUEZ, R. PhD
}

\section{The paradox of being physically active but sedentary or sedentary but physically active}

The use of objective methods to measure physical activity and thus assess sedentary lifestyle, may change the definition of individuals as being sedentary or physically active. This situation would also interfere on the benefits of physical activity and the dangerous effects of sedentary lifestyle on health. The aim of this review is to clarify the changes that have recently occurred in this area. These could be included in future health care strategies and recommendations for the population.

(Rev Med Chile 2014; 142: 72-78)

Key words: Health care; Motor activity; Sedentary lifestyle.
L os cambios económicos, urbanísticos, tecnológicos, entre otros, acontecidos en las últimas décadas, han generado modificaciones importantes en los hábitos y estilo de vida de nuestra sociedad ${ }^{1}$. Como resultado de esta transición social se han reducido drásticamente los niveles de actividad física de las personas, lo que se ha asociado con un aumento sostenido del sobrepeso y de la obesidad, como también de las enfermedades crónicas no transmisibles, lo que ha elevado considerablemente el riesgo cardiometabólico, como la mortalidad por estas causas en la población ${ }^{2}$. La OMS señala a la inactividad física como el cuarto factor de riesgo más importante de mortalidad en todo el mundo (6\% de defunciones a nivel mundial), superándola sólo la presión arterial elevada (13\%), el consumo de tabaco (9\%) y la glicemia elevada $(6 \%)^{3}$.

En contraparte, realizar tanto actividad física de manera regular como ejercicio físico -este último difiere del primero en su clara intencionalidad y sistematicidad ${ }^{4}$ - genera una diversidad de adaptaciones a nivel muscular, óseo, metabólico como respiratorio y cardiovascular ${ }^{5}$, mejorando de esta manera el estado de salud de las personas ${ }^{6,7,8}$. Asimismo, existe fuerte evidencia científica respecto a los beneficios del ejercicio físico sobre la patogenia de diversas enfermedades ${ }^{9,10}$, llegando al punto de describir al ejercicio como la píldora ideal para mejorar la salud, debido principalmente a su efecto multiorgánico a diferencia de los medicamentos ${ }^{11}$. Cabe señalar que para lograr dichos beneficios parece existir un claro efecto dosis/respuesta, que dependería tanto del tiempo como de la intensidad del ejercicio ${ }^{10,12}$. Mientras mayores sean los niveles de actividad física de la población, menor será la probabilidad de mortalidad global ${ }^{13,14}$.

Actualmente, se recomienda a los niños y adolescentes acumular al menos $60 \mathrm{~min}$ de actividad física aeróbica de moderada a vigorosa intensidad cada día ${ }^{15-19}$. Para la población adulta se recomiendan al menos 150 min de actividad 
física de intensidad moderada a vigorosa o al menos 75 min de actividad física vigorosa por semana, pudiendo ser acumulada en tandas de 10 min de actividad continua ${ }^{15,19,20,21,22}$, como por ejemplo correr, nadar, caminar rápido, bailar, andar en bicicleta, etc. ${ }^{23}$. A los adultos mayores se les recomienda realizar actividad física de 30 a 60 min de intensidad moderada al día. Las actividades de intensidad vigorosa se tratan de evitar en estas personas debido a que presentan un alto riesgo de lesión y baja adherencia ${ }^{19,24-26}$.

No obstante lo anterior, la mayor parte de la población chilena no cumple estas recomendaciones de actividad física. Evidencia de esto es el elevado nivel de sedentarismo presente tanto en la población adulta $(88,6 \%)$ como en la de niños y jóvenes entre 15 y 19 años $(76,9 \%)^{27}$, y la alta prevalencia de sobrepeso y obesidad tanto en la población preescolar y escolar ${ }^{28,29}$ como adulta ${ }^{27}$. Estas cifras podrían agravarse aún más si consideramos que "demasiado tiempo sedente no es lo mismo que poco activo físicamente".

\section{Relación entre conducta sedentaria y actividad física}

Se ha demostrado que pasar demasiado tiempo sedente (acostado en cama, sentado, conduciendo, leyendo o destinar poco tiempo a actividades ambulatorias) reduciría rápidamente la salud metabólica ${ }^{30}$. Siendo esto asociado tanto a anormalidades en el metabolismo de la glucosa ${ }^{31,32}$, como al síndrome metabólico ${ }^{33}$, afectando especialmente a las personas que ven más de $4 \mathrm{~h}$ de televisión al día. Asimismo, esta conducta ha sido relacionada con un aumento importante en el riesgo de muerte por enfermedad cardiovascular, diabetes tipo II, mortalidad por todas las causas, cáncer, etc. ${ }^{34,36-38}$.

Durante los últimos años, los estudios han mejorado significativamente la calidad de las investigaciones en esta área ${ }^{39}$, empleando la acelerometría ${ }^{31-34,36,37,39,40-43}$, un método objetivo para cuantificar los niveles e intensidad de la actividad física, dejando de manifiesto las limitaciones inherentes al uso de los cuestionarios para dicha valoración $\mathrm{n}^{39,44}$.

Diversas investigaciones que han empleado esta herramienta han demostrado que independiente de los niveles de actividad física, existe una relación inversa entre el tiempo destinado a conductas sedentarias y el perímetro de cintura, la presión arterial diastólica y marcadores de riesgo cardiometabólico en mujeres ${ }^{40}$ y niños ${ }^{41}$. Por otra parte, el nivel de adiposidad estaría vinculado con los niveles de actividad física, pero no con los niveles de conducta sedentaria ${ }^{45}$. Lo expuesto deja en evidencia que es necesario comprender que estamos frente a dos campos complementarios pero que estarían ejerciendo sus efectos en la salud posiblemente por vías independientes ${ }^{30,46}$.

\section{Paradoja}

La manera como se ha determinado el nivel de sedentarismo en la población es identificando aquellos individuos que son inactivos físicamen$\mathrm{te}^{47}$. La mayoría de los cuestionarios empleados en las encuestas de salud, incluida la Encuesta Nacional de Salud (ENS) 2009-2010, categorizan a una persona como "activa" si cumple la recomendación de realizar al menos 30 min de actividad física 3 veces a la semana, o bien, categorizan a una persona como "sedentaria" si no cumple dicho parámetro ${ }^{27}$.

$\mathrm{Al}$ respecto, la última ENS de Chile, indica que $88,6 \%$ de la población adulta es sedentaria ${ }^{27}$. Sin embargo, esto podría tener una doble lectura si consideramos las nuevas publicaciones en esta materia $^{39,42}$. Por un lado, $11,4 \%$ de la población de nuestro país que es activa físicamente podría presentar una elevada conducta sedentaria; por otro lado, la población sedentaria podría presentar altos niveles de actividad física de intensidad ligera.

Sumado a lo anterior, ha surgido nueva evidencia respecto a un verdadero "efecto de interferencia" entre los beneficios de cumplir con las recomendaciones de actividad física, los que se verían afectados por la conducta sedentaria ${ }^{39}, y$ los efectos perjudiciales en la salud que presentan las personas sedentarias los que podrían mejorar si éstas realizan actividad física regular de intensidad ligera $^{42}$ (Figura 1). Lo expuesto hasta ahora genera un cambio importante en la noción sobre quién es realmente una persona sedentaria y activa físicamente.

\section{Activo físicamente pero con hábito sedentario: efectos en la salud}

El equipo de trabajo de Hamilton ha publicado una serie de artículos en los que han demostrado que los efectos fisiológicos y moleculares de dema- 


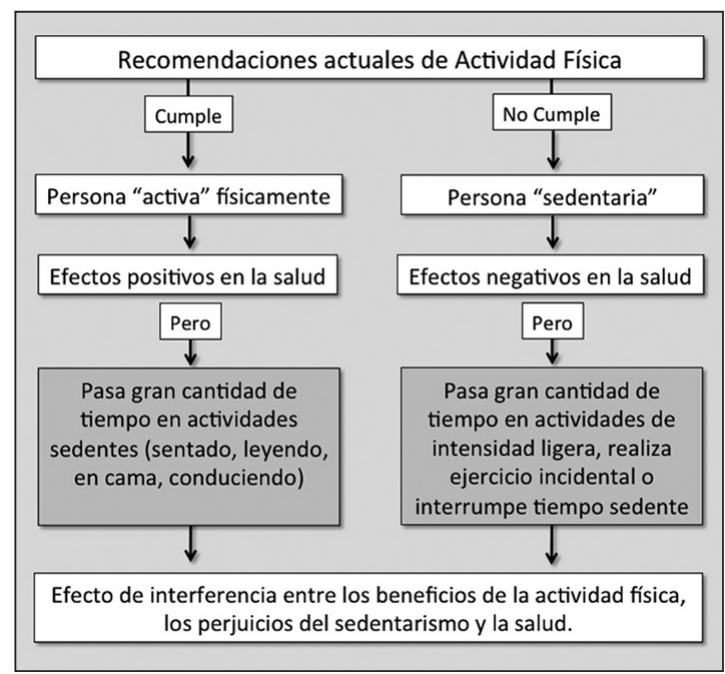

Figura 1. Efecto de interferencia entre los beneficios de la actividad física, los perjuicios del sedentarismo y la salud.

siado tiempo sedentario parecen ser diferentes a los observados con demasiado tiempo sin actividad física ${ }^{48-51}$. Además, otros estudios han señalado que a pesar de cumplir con las recomendaciones de actividad física, destinar mucho tiempo a actividades de tipo sedentarias, podría interferir sobre los efectos beneficiosos del ejercicio en la salud $^{42,52}$ (Figura 1).

En niños, se demostró que pasar demasiado tiempo viendo televisión posee una relación inversa con los biomarcadores cardiometabólicos, siendo esta asociación independiente de sus niveles de actividad física ${ }^{40}$. Haely y su grupo de investigación, en el año $2008^{53}$, concluyeron que independiente del tiempo empleado en actividades físicas de moderada a vigorosa intensidad, el tiempo sedentario presenta una asociación significativa con el perímetro de cintura en adultos, y en una muestra de adultos mayores se determinó que, independiente de cumplir con los niveles de actividad física recomendados para esta población, mientras menos tiempo se pasa frente al televisor menor era la prevalencia de sobrepeso y obesidad ${ }^{2}$. Estas investigaciones confirmarían que la relación entre los niveles de conducta sedentaria y mortalidad se mantendrían incluso en las personas físicamente activas $^{37}$.

En un reciente artículo de Dunstan y colaboradores en el año $2012^{54}$, señalan que tras analizar la evidencia científica de sus estudios experimentales y epidemiológicos han llegado a la conclusión de que mucho tiempo sentado debería ser considerado como un componente independiente de los niveles de actividad física de las personas. Por tanto, así como es importante incrementar el tiempo destinado a la actividad física para mejorar la salud metabólica, también lo es disminuir el tiempo en actividades sedentarias ${ }^{53}$.

\section{Sedentario pero activo físicamente: actividad incidental y continuum de intensidad}

Hasta la fecha, se ha establecido la importancia tanto del tiempo como de la intensidad de la actividad física para lograr una diversidad de beneficios en la salud ${ }^{10,55}$. Considerando el planteamiento anterior, la mayoría de las recomendaciones sugieren que es necesario alcanzar un umbral mínimo (entre $40 \%$ y $50 \%$ de la frecuencia cardiaca de reserva o una intensidad $>3$ METs) y un tiempo mínimo de actividad física continua (10 $\mathrm{min}$ ) para alcanzar dichos efectos beneficiosos ${ }^{22,56}$. Un ejemplo de esto, es que si se logra un incremento de 1 MET (equivalente metabólico) en la condición física, se podría apreciar una disminución de $13 \%$ y $15 \%$ en la mortalidad global y enfermedad cardiovascular respectivamente ${ }^{39}$.

Contrario al planteamiento anterior, un reciente estudio de McGuire y Ross, 2011, en personas que no cumplían con las recomendaciones de actividad física, demostró que si estas destinaban gran parte de su tiempo a actividades de intensidad ligera ( $<3 \mathrm{METs}$ ), como por ejemplo las labores del hogar ${ }^{23}$, de igual manera se generaban cambios positivos en la condición física ${ }^{39}$. Además se apreció que si la actividad física de intensidad moderada a vigorosa (>3 METs) se realizaba de manera "incidental" (entendiendo ésta como la actividad física no programada que dura menos de $10 \mathrm{~min}$ y que se acumula en tandas discontinuas), también aumentaba la capacidad cardiorrespiratoria.

Por una parte, estos hallazgos, además de diferir bastante de las recomendaciones actuales de actividad física ${ }^{15-22,24-26}$, ponen en duda la existencia de un umbral mínimo para mejorar la salud con ejercicio o actividad física, y por otra, inducen a pensar que existiría un "continuum" relacionado con la intensidad de la actividad física, demostrando que tanto las actividades de intensidad ligera, pasando por las moderadas hasta llegar a 
las vigorosas, generarían beneficios en la salud y podrían disminuir la mortalidad de la población ${ }^{42}$. Por último, son necesarios más estudios para aumentar la evidencia al respecto y para corroborar dicho planteamiento.

\section{Beneficios de interrumpir el hábito sedentario}

En una revisión de Saunders y colaboradores del año 2012 se ha descrito que incluso períodos de inactividad menores a 7 días pueden incrementar el riesgo metabólico ${ }^{57}$. Pero por otro lado, se ha demostrado que las personas que interrumpen repetidamente su tiempo sedente (pasando de posición sentado a de pie, o bien, estando de pie y comenzar a caminar) tienen un menor perímetro de cintura, menor índice de masa corporal, menores niveles de triglicéridos y glucosa plasmática después de 2 h. Además, al dividir la muestra en cuartiles, se observa que el cuartil que más veces interrumpía el tiempo sedentario tenía $6 \mathrm{~cm}$ menos de perímetro de cintura en comparación al que menos veces lo hacia ${ }^{58}$.

Otro reciente estudio demostró que las personas con sobrepeso y obesidad que interrumpían cada 20 min su hábito sedentario caminando 2 min a ligera o moderada intensidad disminuyeron la glucosa postprandial y los niveles de insulina. Esta podría ser una importante estrategia tanto a nivel clínico como a nivel de salud pública para reducir el riesgo cardiovascular ${ }^{59}$. Por tanto, la evidencia al respecto pareciera indicar que la manera como se acumula el volumen total del tiempo sedentario, sería una variable que también estaría relacionada con la salud de las personas ${ }^{1,60,61}$ (Figura 1).

Tabla 1. Cuadro resumen, puntos claves y recomendaciones

- Cumplir con las recomendaciones actuales de actividad física conlleva grandes beneficios sobre la salud y disminución de la mortalidad de la población

- A mayor cantidad de tiempo e intensidad del ejercicio o actividad física mayores parecen ser los beneficios en la salud

- Se debería comprender que "Demasiado tiempo sedente no es lo mismo que poco activo físicamente", y que sus efectos podrían ser independientes entre si

- Una persona activa físicamente podría presentar un elevado hábito sedentario

- Una persona sedentaria podría presentar un nivel elevado de actividad física de intensidad ligera

- La actividad física de intensidad ligera también ha demostrado mejorar la capacidad cardiorrespiratoria

- La actividad física de moderada a alta intensidad realizada de manera incidental, en tandas discontinuas de menos de 10 min, también ha demostrado mejorar la capacidad cardiorrespiratoria

- Se debería reducir el tiempo frente a la televisión, acostado y conduciendo entre otras actividades similares de bajo costo energético

- Interrumpir el hábito sedentario, parándose y caminar tan sólo 2 min, cada 20 min, podría ser una estrategia para generar beneficios en la salud

- La manera como se acumula y no sólo el volumen total del tiempo sedentario sería una variable que también estaría relacionada con la salud de las personas

- Existiría un continuum relacionado con la intensidad del ejercicio o actividad física y los beneficios sobre la salud

- Además de seguir promoviendo el aumento de los niveles e intensidad de la actividad física en la población se deberían incluir en las Estrategia de Salud ciertas recomendaciones, tales como: disminuir el tiempo destinado a ver televisión, estar acostado o conduciendo; interrumpir continuamente el hábito sedentario; aumentar el tiempo destinado durante el día a actividades de intensidad ligera

- Se podría mejorar la calidad de los estudios en el área del hábito sedentario y de la actividad física empleando métodos más objetivos para la cuantificación de los niveles e intensidad de estos, como es el caso de la acelerometría

- Las futuras investigaciones deberían distinguir claramente las diferencias terminológicas entre ser sedentario e inactivo físicamente

- Se debería incrementar el número de investigaciones en esta área de estudio, sobre todo los de tipo longitudinales y experimentales 


\section{Consenso: ¿sedentario o inactivo físicamente?}

Actualmente, en la literatura es común apreciar que los términos "inactivo físicamente" $y$ "sedentario" se utilizan para definir lo mismo, a pesar de que la evidencia científica viene demostrando que deberían ser considerados como conceptos independientes uno del otro ${ }^{34,35,37,38,40}$. Por lo tanto, se hace necesario definirlos con la finalidad de distinguir en las futuras investigaciones, las características del tipo de población o grupo control que se está estudiando. Tremblay y colaboradores en el año 2012 proponen que, el "hábito o conducta sedentaria" sea definido como cualquier comportamiento con un gasto metabólico menor a 1,5 METs. Por el contrario, se sugiere que los autores empleen el término "inactivo físicamente" para describir a las personas que no cumplan con las recomendaciones mínimas de actividad física ${ }^{46}$.

Todos los antecedentes descritos anteriormente en este artículo de revisión resaltan la necesidad de incluir nuevas recomendaciones sobre actividad física y disminución e interrupción del hábito sedentario con la finalidad de mejorar la salud de la población; además de incentivar las investigaciones con métodos más objetivos y aclarar las diferencias terminológicas existentes sobre esta materia (Tabla 1).

\section{Conclusiones}

Durante los últimos años diversas publicaciones han dejado de manifiesto que los efectos sobre la salud, al permanecer demasiado tiempo sedentario como también ser poco activo físicamente, podrían estar actuando por vías independientes. Demostrando además, que es fundamental discriminar entre una persona sedentaria y otra inactiva físicamente, y que la acelerometría se ha convertido en una herramienta fundamental para mejorar la calidad de las investigaciones en esta materia. Los nuevos antecedentes sobre actividad física y conducta sedentaria reafirman la necesidad de incluir nuevas recomendaciones en las estrategias de salud para la población, y además resaltan la importancia de potenciar este nuevo foco de investigación.

Agradecimientos: Se agradece al Director de la Carrera de Pedagogía en Educación Física de la Universidad Viña del Mar, Sr. Ramón Baron- ti Barella, la docente Sra. Lilian Castro y Evita Skinski por sus valiosos aportes y correcciones al manuscrito.

\section{Referencias}

1. Owen N, Healy GN, Matthews CE, Dunstan DW. Too much sitting: the population health science of sedentary behavior. Exerc Sport Sci Rev 2010; 38: 105-13.

2. Inoue S, Sugiyama T, Takamiya T, Oka K, Owen N, Shimomitsu T. Television viewing time is associated with overweight/obesity among older adults, independent of meeting physical activity and health guidelines. J Epidemiol 2012; 22: 50-6.

3. OMS. Global health risks: mortality and burden of disease attributable to selected major risks. 2009.

4. Caspersen CJ, Powell KE, Christenson GM. Physical activity, exercise, and physical fitness: definitions and distinctions for health-related research. Public Health Rep 1985; 10: 126-31.

5. Pérez AB. [Exercise as the Cornerstone of Cardiovascular Prevention). Rev Esp Cardiol 2008; 61: 514-28.

6. Gleeson M, Bishop NC, Stensel DJ, Lindley MR, Mastana SS, Nimmo MA. The anti-inflammatory effects of exercise: mechanisms and implications for the prevention and treatment of disease. Nat Rev Immunol 2011; 11: 607-15.

7. Rodríguez J, Márquez S, de Abajo S. Sedentarismo y salud: efectos beneficiosos de la actividad física. Apunts Educación física y deportes 2006; 83: 12-24.

8. Chomistek AK, Chiuve SE, Jensen MK, Cook NR, Rimm EB. Vigorous physical activity, mediating biomarkers, and risk of myocardial infarction. Med Sci Sports Exerc 2011; 43: 1884-90.

9. Pedersen BK, Saltin B. Evidence for prescribing exercise as therapy in chronic disease. Scand J Med Sci Sports 2006; 16 Suppl 1:3-63.

10. Subirats E, Subirats G, Soteras I. Prescripción de ejercicio físico: indicaciones, posología y efectos adversos. Med Clin 2012; 138: 18-24.

11. Goodyear LJ. The exercise pill too good to be true? N Engl J Med 2008; 359: 1842-4.

12. Leggate M, Carter WG, Evans MJ, Vennard RA, SribalaSundaram S, Nimmo MA. Determination of inflammatory and prominent proteomic changes in plasma and adipose tissue after high-intensity intermittent training in overweight and obese males. J Appl Physiol 2012; 112: 1353-60.

13. Paffenbarger Jr RS, Kampert JB, Lee IM, Hyde RT, Leung RW, Wing AL. Changes in physical activity and other 
lifeway patterns influencing longevity. Medicine and Science in Sports and Exercise 1994; 26: 857-65.

14. Andersonn JJB. The important role of physical activity in skeletal development: how exercise may counter low calcium intake. American Journal of Clinical Nutrition 2000; 71: 1384-86.

15. O'Donovan G, Blazevich AJ, Boreham C, Cooper AR, Crank H, Ekelund U, et al. The ABC of Physical Activity for Health: a consensus statement from the British Association of Sport and Exercise Sciences. J Sports Sci 2010; 28: 573-91.

16. Laure P, Mangin G. Advising parents on physical activity for children between 0 and 5 years. J Sports Med Phys Fitness 2011; 51: 467-72.

17. Graham I, Atar D, Borch-Johnsen K, Boysen G, Burell G, Cifkova R, et al. Expert Panel on Integrated Guidelines for Cardiovascular Health and Risk Reduction in Children and Adolescents: Summary Report. Pediatrics 2011; 128: S213-S256.

18. Skouteris H, Dell'Aquila D, Baur LA, Dwyer GM, McCabe MP, Ricciardelli LA, et al. Physical activity guidelines for preschoolers: a call for research to inform public health policy. Med J Aust 2012; 196: 174-7.

19. OMS. Global Recommendations on Physical Activity for Health. 2010.

20. Garber CE, Blissmer B, Deschenes MR, Franklin BA, Lamonte MJ, Lee I-M, et al. American College of Sports Medicine position stand. Quantity and quality of exercise for developing and maintaining cardiorespiratory, musculoskeletal, and neuromotor fitness in apparently healthy adults: guidance for prescribing exercise. Med Sci Sports Exerc 2011; 43: 1334-59.

21. Graham I, Atar D, Borch-Johnsen K, Boysen G, Burell G, Cifkova R, et al. European guidelines on cardiovascular disease prevention in clinical practice: executive summary: Fourth Joint Task Force of the European Society of Cardiology and Other Societies on Cardiovascular Disease Prevention in Clinical Practice (Constituted by representatives of nine societies and by invited experts). Eur Heart J 2007; 28: 2375-414.

22. Haskell WL, Lee I-M, Pate RR, Powell KE, Blair SN, Franklin BA, et al. Physical activity and public health: updated recommendation for adults from the American College of Sports Medicine and the American Heart Association. Circulation 2007; 116: 1081-93.

23. Ainsworth BE, Haskell WL, Herrmann SD, Meckes N, Bassett DR Jr, Tudor-Locke C, et al. 2011 Compendium of Physical Activities: a second update of codes and MET values. Med Sci Sports Exerc 2011; 43: 1575-81.

24. Chodzko-Zajko WJ, Proctor DN, Fiatarone Singh MA, Minson CT, Nigg CR, Salem GJ, et al. American College of Sports Medicine position stand. Exercise and physical activity for older adults. Med Sci Sports Exerc 2009; 41: 1510-30.

25. Nelson ME, Rejeski WJ, Blair SN, Duncan PW, Judge JO, King AC, et al. Physical activity and public health in older adults: recommendation from the American College of Sports Medicine and the American Heart Association. Med Sci Sports Exerc 2007; 39: 1435-45.

26. Elsawy B, Higgins KE. Physical activity guidelines for older adults. Am Fam Physician 2010; 81: 55-9.

27. ENS, Encuesta Nacional de Salud, 2009-2010. Ministerio de Salud de Chile. www.redsalud.gov.cl/ Visitada 05/06/2012.

28. JUNAEB, Junta Nacional de Auxilio Escolar y Becas, 2003; Disponible en: http://www.junaeb.cl/prontus_junaeb/site/edic/base/port/inicio.html Visitado 20/01/2012.

29. Eyzaguirre CF, Mericq GV, Ceresa OS, Youlton RR, Zacarías SJ. Prevalencia de sobrepeso y obesidad en niños que se controlan en pediatría ambulatoria en Clínica Las Condes. Rev Chil Pediatr 2005; 76: 143-9.

30. Thyfault JP, Booth FW. Lack of regular physical exercise or too much inactivity. Curr Opin Clin Nutr Metab Care 2011; 14: 374-8.

31. Dunstan DW, Salmon J, Healy GN, Shaw JE, Jolley D, Zimmet PZ, et al. Association of television viewing with fasting and 2-h postchallenge plasma glucose levels in adults without diagnosed diabetes. Diabetes Care 2007; 30: 516-22.

32. Dunstan DW, Salmon J, Owen N, Armstrong T, Zimmet PZ, Welborn TA, et al. Physical activity and television viewing in relation to risk of undiagnosed abnormal glucose metabolism in adults. Diabetes Care 2004; 27: 2603-9.

33. Dunstan DW, Salmon J, Owen N, Armstrong T, Zimmet PZ, Welborn TA, et al. Associations of TV viewing and physical activity with the metabolic syndrome in Australian adults. Diabetologia 2005; 48: 2254-61.

34. Dunstan DW, Barr EL, Healy GN, Salmon J, Shaw JE, Balkau B, et al. Television viewing time and mortality: the Australian Diabetes, Obesity and Lifestyle Study (AusDiab). Circulation 2010; 121: 384-91.

35. Grøntved A, Hu FB. Television viewing and risk of type 2 diabetes, cardiovascular disease, and all-cause mortality. JAMA 2011; 305: 2448-55.

36. Warren TY, Barry V, Hooker SP, Sui X, Church T, Blair $\mathrm{SN}$. Sedentary behaviors increase risk of cardiovascular disease mortality in men. Med. Sci. Sports Exerc 2010; 42: 879-85.

37. Katzmarzyk PT, Church TS, Craig CL, Bouchard C. Sitting time and mortality from all causes, cardiovascular 
disease, and cancer. Med Sci Sports Exerc 2009; 41: 998 1005.

38. Thorp AA, Owen N, Neuhaus M, Dunstan DW. Sedentary behaviors and subsequent health outcomes in adults: a systematic review of longitudinal studies, 1996-2011. Am J Prev Med 2011; 41: 207-15.

39. McGuire KA, Ross R. Incidental physical activity is positively associated with cardiorespiratory fitness. Med Sci Sports Exerc 2011; 43: 2189-94.

40. Wijndaele K, Healy GN, Dunstan DW, Barnett AG, Salmon J, Shaw JE, et al. Increased cardio-metabolic risk is associated with increased TV viewing time. Med Sci Sports Exerc 2010; 21; 8: 98.

41. Ekelund U, Brage S, Froberg K, Harro M, Anderssen SA, Sardinha LB, et al. TV viewing and physical activity are independently associated with metabolic risk in children: the European Youth Heart Study. PLoS Med 2006; 3: e488.

42. Hsu YW, Belcher BR, Ventura EE, Byrd-Williams CE, Weigensberg MJ, Davis JN, et al. Physical activity, sedentary behavior, and the metabolic syndrome in minority youth. Med Sci Sports Exerc 2011; 43: 2307-13.

43. Martínez-Gómez D, Eisenmann JC, Gómez-Martínez S, Veses A, Marcos A, Veiga OL. Sedentary behavior, adiposity and cardiovascular risk factors in adolescents. The AFINOS study. Rev Esp Cardiol 2010; 63: 277-85.

44. Shephard RJ. Limits to the measurement of habitual physical activity by questionnaires. Br J Sports Med 2003; 37: 197-206.

45. Chaput JP, Lambert M, Mathieu ME, Tremblay MS, O' Loughlin J, Tremblay A. Physical activity vs sedentary time: independent associations with adiposity in children. Pediatr Obes 2012; 7: 251-8.

46. Tremblay MS, Colley RC, Saunders TJ, Healy GN, Owen N. Physiological and health implications of a sedentary lifestyle. Appl Physiol Nutr Metab 2010; 35: 725-40.

47. Buhring K, Oliva P, Bravo C. Determinación no experimental de la conducta sedentaria en escolares. Rev Chil Nutr 2009; 36: 23-30.

48. Bey L, Hamilton MT. Suppression of skeletal muscle lipoprotein lipase activity during physical inactivity: a molecular reason to maintain daily low-intensity activity. J Physiol 2003; 551: 673-82.

49. Hamilton MT, Hamilton DG, Zderic TW. Exercise physiology versus inactivity physiology: An essential concept for understanding lipoprotein lipase regulation. Exerc Sport Sci Rev 2004; 32: 161-6.
50. Hamilton MT, Hamilton DG, Zderic TW. Role of low energy expenditure and sitting in obesity, metabolic syndrome, type 2 diabetes, and cardiovascular disease. Diabetes 2007; 56: 2655-67.

51. Hamilton MT, Healy GN, Dunstan DW, Zderic TW, Owen N. Too little exercise and too much sitting: Inactivity physiology and the need for new recommendations on sedentary behavior. Curr Cardiovasc Risk Rep 2008; 2: $292-8$

52. Healy G, Owen N. Conducta sedentaria y biomarcadores del riesgo cardiometabólico en adolescentes: un problema científico y de salud pública emergente. Rev Esp Cardiol 2010; 63: 261-4.

53. Healy GN, Wijndaele K, Dunstan DW, Shaw JE, Salmon J, Zimmet PZ, et al. Objectively measured sedentary time, physical activity, and metabolic risk: the Australian Diabetes, Obesity and Lifestyle Study (AusDiab). Diabetes Care 2008; 31:369-71.

54. Dunstan DW, Howard B, Healy GN, Owen N. Too much sitting - A health hazard. Diabetes Res Clin Pract 2012; 38: 105-13.

55. Mendes R, Sousa N, Themudo Barata JL. Physical activity and public health: recommendations for exercise prescription. Acta Med Port 2011; 24: 1025-30.

56. Norton K, Norton L, Sadgrove D. Position statement on physical activity and exercise intensity terminology. J Sci Med Sport 2010; 13: 496-502.

57. Saunders TJ, Larouche R, Colley RC, Tremblay MS. Acute Sedentary Behaviour and Markers of Cardiometabolic Risk: A Systematic Review of Intervention Studies. J Nutr Metab 2012; 2012: 712435.

58. Healy GN, Dunstan DW, Salmon J, Cerin E, Shaw JE, Zimmet PZ, Owen N. Breaks in sedentary time: beneficial associations with metabolic risk. Diabetes Care 2008; 31 : 661-6.

59. Dunstan DW, Kingwell BA, Larsen R, Healy GN, Cerin E, Hamilton MT, Shaw JE, Bertovic DA, Zimmet PZ, Salmon J, Owen N. Breaking up prolonged sitting reduces postprandial glucose and insulin responses. Diabetes Care 2012; 35: 976-83.

60. Bankoski A, Harris TB, McClain JJ, Brychta RJ, Caserotti P, Chen KY, Berrigan D, Troiano RP, Koster A. Sedentary activity associated with metabolic syndrome independent of physical activity. Diabetes Care 2011; 34: 497-503.

61. Chastin SF, Granat MH. Methods for objective measure, quantification and analysis of sedentary behaviour and inactivity. Gait Posture 2010; 31: 82-6. 